\title{
Connecting users to trusted geospatial information for Europe
}

\author{
M. J. Cory \\ EuroGeographics AISBL, mick.cory@eurogeographics.org
}

Keywords: Map production and geo-information management, location based services, topographic mapping

\begin{abstract}
:
In recent years, we have seen an explosion in location-based services. Whether it is maps, cadastral data or land registration, geospatial information is driving applications to realise social, economic and environmental benefits for us all. Yet in the age of 'Big Data' and the 'Internet of Things', how can we know which data sources are reliable and how easy is it to find accurate, high-quality and detailed information that we can trust? Knowing that reliable sources exist, and where to obtain them, is essential for governments and those making critical decisions that affect all our lives
\end{abstract}

As the official bodies responsible for national cadastre, land registration, geodetic surveying and mapping activities in Europe, European national mapping and cadastral agencies (NMCAs) fulfil an essential role providing definitive and detailed geospatial information. In an ever-changing world, NMCAs play an important and often critical role in helping to address the key global and regional issues that affect society, such as climate change, sustainable development, a digital economy, migration, security and health. With these challenges extending beyond national borders, society now expects a borderless digital economy and property market, as well as fully connected national databases for stronger cross-border emergency planning and environmental monitoring.

As their membership association, EuroGeographics is committed to supporting European NMCAs as they improve access to their rich source of trusted, authoritative geospatial information.

EuroGeographics is an independent international not-for-profit organisation representing Europe's National Mapping, Cadastral and Land Registration Authorities. We believe in a society empowered by the use of trusted geospatial services from these official national sources. EuroGeographics strength lies in our extensive membership and we are proud to represent more than 60 organisations from 46 countries covering the whole of geographical Europe. We deliver benefits for each regardless of the geographical, technical, political, organisational, linguistic and business parameters in which they work. We support the public good by representing our members' interests, maintaining networks that help our members improve their capabilities and role, and by facilitating access to and use of our members' geospatial data and services.

EuroGeographics' has the strategic objective of facilitating access to our members' authoritative data for international users of harmonised, pan-European, geospatial information and services. It coordinates the compilation and production of pan-European datasets, and has been active in exploring the development of online services. The Open European Location Services (Open ELS) project has developed services to demonstrate and test the level of interest in pan-European authoritative geospatial information. EuroGeographics has coordinated this two-year project which was co-financed by the European Union's Connecting Europe Facility working with partners from member organisations in Finland, Germany, Great Britain, Norway, Poland, Spain, Sweden and The Netherlands. The Project has focused on facilitating access to, and encouraging the take up and use of this information, and is strongly user orientated with a programme of activities to support the digital economy.

As well as test services, an Open ELS data policy has been developed which applies only to the geospatial pan-European data and services developed and maintained by EuroGeographics and its members through the Open ELS Project. The Policy was drafted using research which revealed the scope of open geospatial data from official national sources across Europe, and revealed the diversity of policy, business and legal approaches across Europe to what is 'open' data.

Additional research, carried out by Deloitte and EuroGeographics, found that small and medium sized enterprises (SMEs) in Europe have a strong appetite for more cross-border authoritative data which could help them improve their product and service offerings, save money and become more competitive. The research highlighted four potential benefits from providing harmonised single access to pan-European geospatial information: The possibility to improve existing products and services offering; the possibility to develop new products and services; the reduced time and costs of dealing with different national mapping and cadastral agencies; and the reduced time and costs for acquiring and accessing data.

Key findings from these research activities will be presented, along with an outline of the project, and what it has achieved. 